\title{
THE BEPICOLOMBO MERCURY PLANETARY ORBITER (MPO) SOLAR ARRAY DESIGN, MAJOR DEVELOPMENTS AND QUALIFICATION
}

\author{
A. Loehberg ${ }^{(1)}$, C. Gruenwald ${ }^{(1)}$, J. Birkel (1), A. Brandl (1), N. Kuebler ${ }^{(1)}$, H. Perrin ${ }^{(1)}$, T. Andreev ${ }^{(1)}$, \\ U. Schuhmacher ${ }^{(2)}$, S. Taylor ${ }^{(3)}$ \\ (1) Airbus DS GmbH, 81663 Munich, Germany \\ (2) Airbus DS GmbH, 88039 Friedrichshafen, Germany \\ (3) ESA/ESTEC, Keplerlaan 1, 2200AG Noordwijk, The Netherlands
}

\begin{abstract}
The BepiColombo Mercury Planetary Orbiter (MPO) solar array has been designed to guarantee the operational requirements in a very severe environment, mainly characterized by high temperatures and high light intensity. Furthermore, the qualification of the MPO solar array requires ca. 5500 thermal cycles.

The final configuration of the solar array is the result of a very complex system analysis aiming at reducing the solar array operative temperatures. The outcome of this was an extensive development and test effort on components which provide sufficiently high temperature resistance and a wing with unique design characteristics.

The MPO solar generator is composed of one wing consisting of three panels and provides an average power output up to $1800 \mathrm{~W}$ during the nominal 1 Earth year mission around Mercury. The wing design is characterised by temperature reduction measures. The flight wing has already passed the majority of the environmental test program.
\end{abstract}

\section{INTRODUCTION}

The BepiColombo mission to planet Mercury is a joint project between the European Space agency (ESA) and JAXA of Japan. Two scientific orbiters, the Mercury Planetary Orbiter (MPO) and the Mercury Magnetospheric Orbiter (MMO) are dedicated to the investigation of the planet.

In Figure 1 the Mercury Composite Spacecraft (MCS) configuration with the Mercury Transfer Module (MTM), MPO and MMO is shown in launch configuration (solar arrays are stowed).

ESA is responsible for the complete MCS system and MTM, MPO plus MOSIF which acts as a sun shield and an interface to the MMO. The MMO satellite design and development is managed by JAXA.



Figure 1. Mercury Composite Spacecraft in stacked configuration - launch configuration

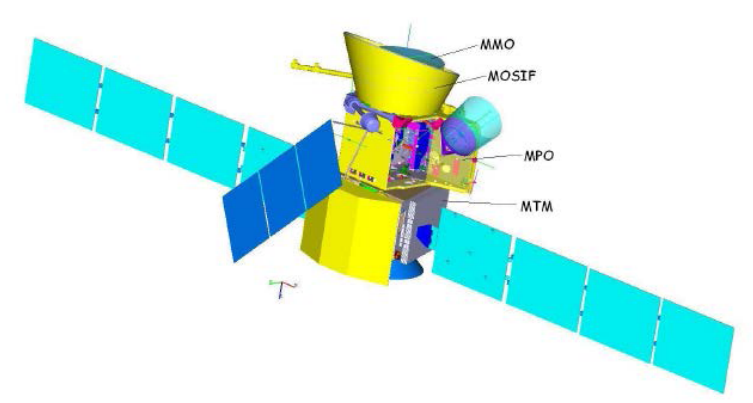

Figure 2. Mercury Composite Spacecraft-cruise configuration

The MTM is used for the transfer to Mercury and hosts an electrical propulsion system with ion thrusters that significantly drive the MTM solar array power requirements. At the end of a 6 years cruise phase to Mercury the MTM is separated from the MCS. After an orbit capture and lowering MMO and MPO are finally separated before the MPO descends to its final orbit. 
As a consequence of the vicinity of planet Mercury to the Sun, the two satellites and the MTM are exposed to extreme light intensities up to 11 solar constants which corresponds to $15.4 \mathrm{~kW} / \mathrm{m}^{2}$. This leads to severe operational conditions of the solar arrays. The MPO solar array is additionally exposed to infrared radiation (between $13.6 \mathrm{~kW} / \mathrm{m}^{2}$ at perihelion and at the subsolar point and $6 \mathrm{~W} / \mathrm{m}^{2}$ at the night side) and the reflection of sun light (albedo $12 \%$ in average but varies considerably) from Mercury's surface. Those boundary conditions result in very high solar array temperatures.

Since 2005 the Airbus DS GmbH Solar Array division has been involved in the development activities related to solar arrays for the MPO and MTM. Concerning the ESA BepiColombo solar arrays, Airbus DS $\mathrm{GmbH}$ Ottobrunn (Munich) is the selected as solar array supplier for the MPO and MTM solar arrays; for the MTM solar array Airbus DS B.V NL has been involved as sub-contractor who is responsible for the MTM overall wing design and assembly. The solar arrays are being procured via Airbus DS GmbH Friedrichshafen who is in the role of the industrial prime for the BepiColombo mission.

In the paper from the ESPC 2011 [1] the evolution of the solar array requirements as a result of technology constraints and major development activities related to high temperature solar arrays are outlined.

The qualification approach for the developed electrical components has been presented in ESPC 2013 [2].

This paper is describing the MPO wing design and qualification activities.

\section{MPO WING DESIGN CHARACTERISTICS}

In the following the MPO wing design characteristics are described.

\subsection{Solar-Mercury Environment and related Orbits as Design Drivers}

Figure 3 shows the Mercury orbit around the Sun and the MPO orbit around Mercury.

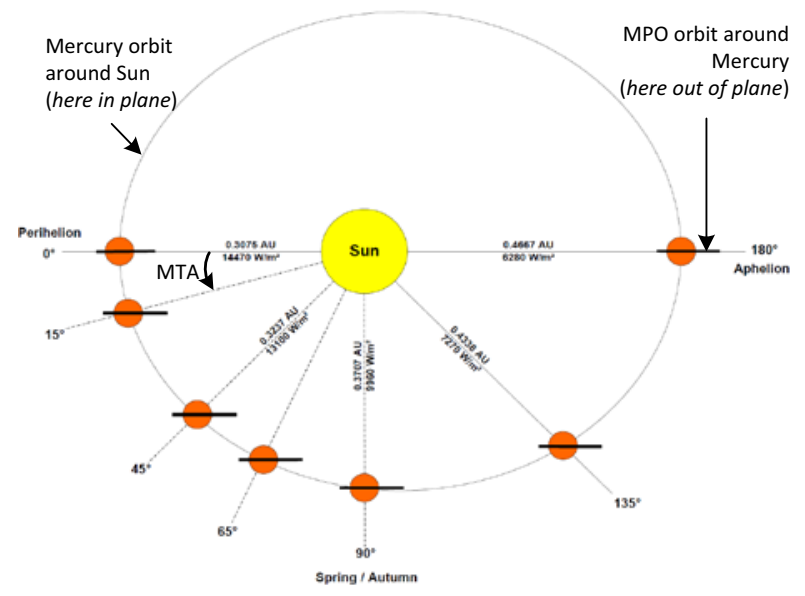

In the following graphic the spacecraft (SC) positions in relation to the planet Mercury and the corresponding Sun direction are visualized for three Mercury true anomalies (MTAs).



Figure 4. Sun direction

The MPO spacecraft (SC) design and the orbits lead to the fact that during the science mission the Sun light, the Mercury infrared heat and it's albedo hit the MPO solar array from many different directions. In addition to this the spacecraft sidewall reflects sun light and emits infrared heat in direction of the solar array. This is visualized by the following graphic.

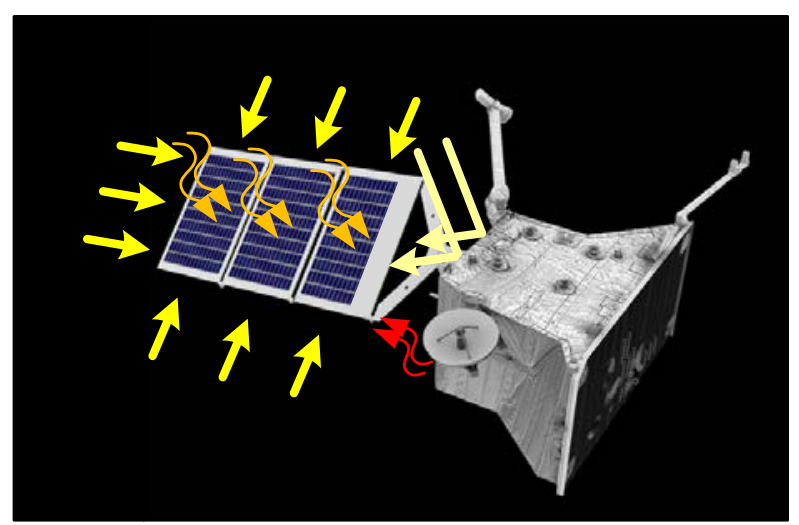

Figure 5. Five "heat sources" from varying directions (graphic copied from [7] and modified)

The variation of intensity and direction of these five "heat sources" during the mission lead to the need that heat protections have to be implemented on all sides of the solar wing.

Figure 5 revealed that solar array operation will be performed under high inclination angles in order to reduce the effective impinging sun light onto the solar array surface. Detailed studies have been performed in order to assess the angle performance of the array $[3,4]$. This is in particular important for precise performance predication under all circumstances.

As also visible in Figure 4 the SC passes several times the Mercury eclipse which leads to the necessity of robustness of the solar array against ca. 5500 thermalcycles down to ca. $-170^{\circ} \mathrm{C}$.

Figure 3. Orbits 


\subsection{OSRs and Kapton-VDA on Front Side}

In between the solar cell strings optical solar reflectors (OSRs) are applied.

The Y-shaped yoke and the first inner quarter of the first panel are fully covered by OSRs instead of solar cells, as due to the hot SC sidewall these areas will become too hot for the chosen and qualified solar cell technology.

At areas with geometrical inhomogeneities on the panel surface Kapton-VDA is used as second surface mirror instead of OSRs.

The following photo shows the wing front side respectively the solar cell and OSR pattern.



Figure 6. MPO solar array front side

For the skins of the sandwich panels ultra-high thermal conducting carbon fibres are chosen to enable a good heat distribution and an efficient cooling of the solar cells by the OSRs. Also for the carbon honeycomb core high conductive carbon fibres are selected to use the panel's rear side as radiator cooler.

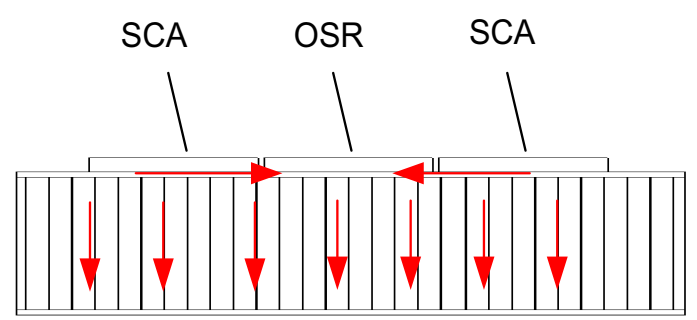

Figure 7. Heat distribution via high conductive fibres

\subsection{OSRs and Kapton-VDA on Rear Side}

Due to reflections of the SC sidewall even the rear side of the yoke and the inner panel are equipped with OSRs. At areas where wires are routed Kapton-VDA is used instead.

The following photo shows the wing rear side.

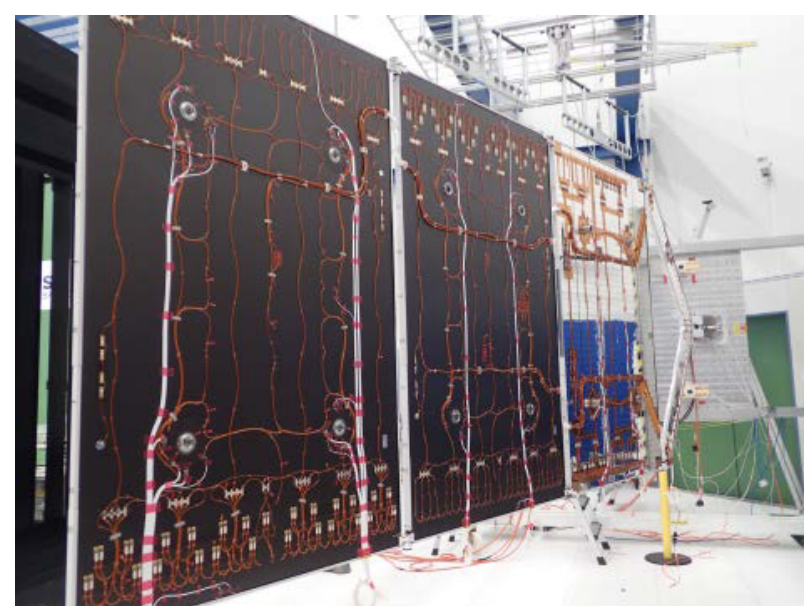

Figure 8. MPO solar array rear side

The next photo shows the rear side of the inner panel in more detail.

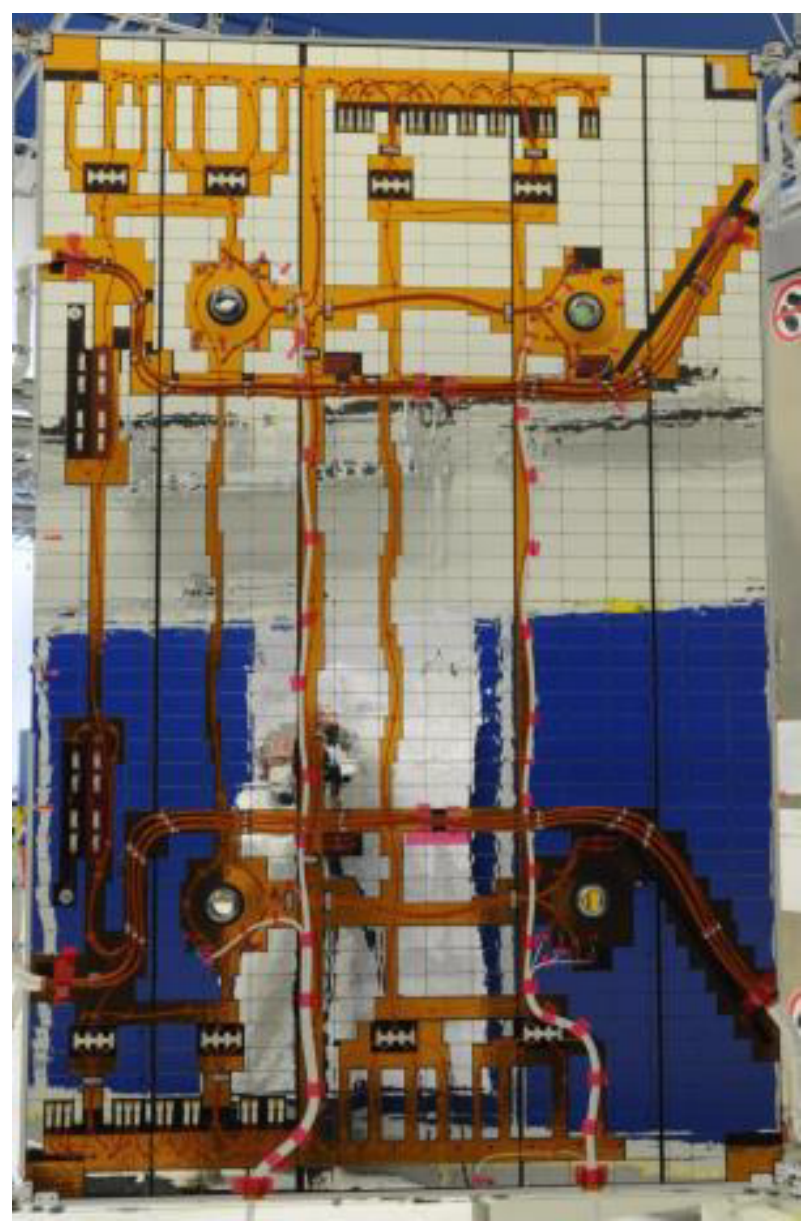

Figure 9. Rear side of inboard panel

2.4 Increase of Solar Aspect Angle and Edge Shields

Despite the usage of the OSRs the temperature of the panels has to be decreased further in order to not exceed the solar cell and substrate qualification temperatures. For this reason the solar panel surface is tilted outwards 
the Sun. By this the solar aspect angle (SAA) is increased.

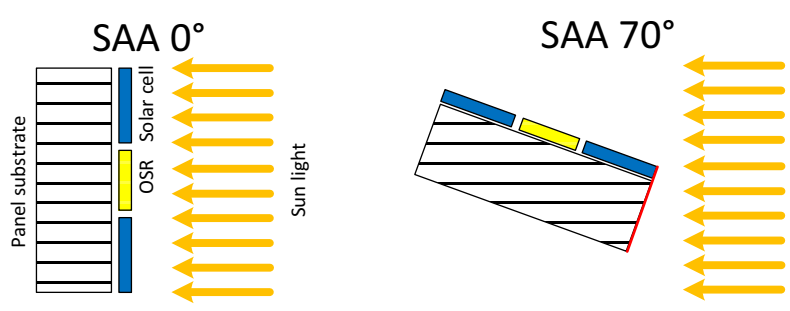

Figure 10. SAA

Drawbacks caused by this are a reduction of the generated electrical power and the need of panel edge shields.

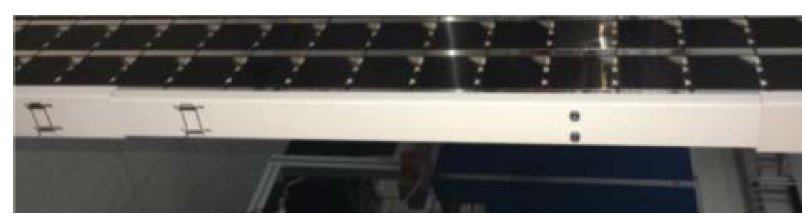

Figure 11. White edge shields

On the Sun averted side the IR emissivity is kept small. Because of the different thermal expansion of the panel substrates and the edge shields, the shields require a bearing with one fix point and one or more sliding/floating points. The predicted temperature of these shields is about $+450^{\circ} \mathrm{C}$.

\section{$2.5 \quad$ Wiring}

Also the electrical wiring turned out to be a very ambitious challenge.

In addition to the five heat sources as shown in Figure 4 the self-heating increases the wire temperatures further. Especially in the vicinity of the hot SC sidewall many measures which decrease the temperatures for one orbit point (e.g. usage of a Sun shield) lead to the opposite effect in another orbit point (e.g. by prevention of IR emittance into deep space).

The solution of this problem was found in a combination of routing the wires on the yoke rear side, usage of sun shields made from white coated Titanium, usage of glass fabrics (Nextel) as heat protection and development of a high temperature electrical insulation $\left(+315^{\circ} \mathrm{C}\right)$ for the wires.

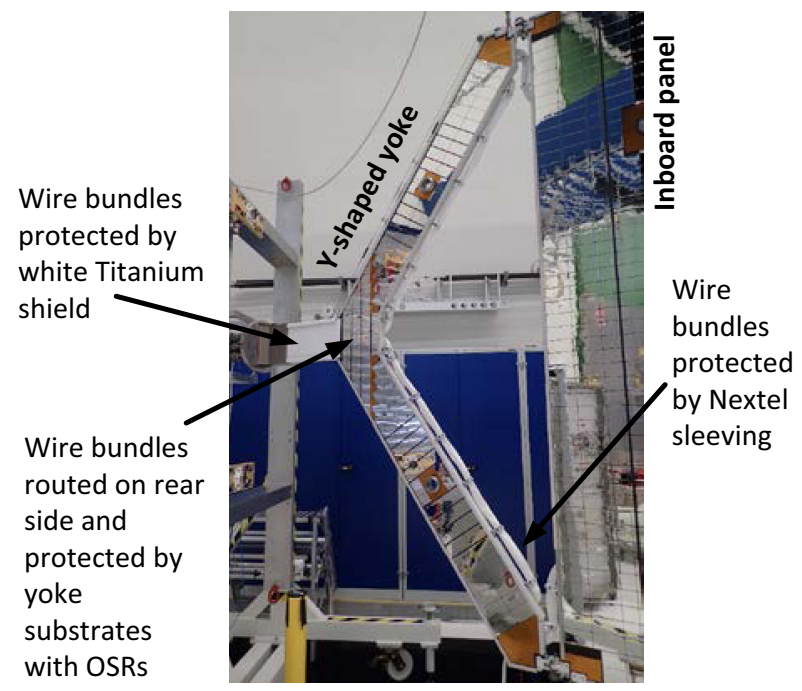

Figure 12. Wire routing at yoke

\subsection{High Temperature Structures}

For the carbon fibre reinforced sandwich panels and yoke substrates the typically used epoxy resins and adhesives were substituted by cyanate based materials which are cured at high temperature. The determination of the correct process parameters which lead to a reliable quality and reproducible mechanical strength was one of the most demanding issues of the project.

Like for most other space structures also for the MPO solar panels is the launch the sizing mechanical load case. Therefore the highest stresses occur at ambient temperatures.

But as the MCS separation events (MTM, MPO, MOSIF and MMO) will occur near Mercury/Sun the structures have to provide a notable mechanical strength also at high temperatures. The solar arrays are already deployed during these events which lead to significant bending moments when the modules are separated. The upper qualification temperature for the substrates is about $+280^{\circ} \mathrm{C}$. The combination of such high temperatures with the mechanical loading was tested on sample level only, not on wing level.

For areas where this temperature is exceeded and for mechanisms Titanium was chosen as base material.

\subsection{Solar Cell Assemblies}

The solar cell assemblies (SCAs) which provide sufficient robustness against high intensity solar light (ca. $11 \mathrm{SC}$ ) in combination with high operating temperatures (up to $+215^{\circ} \mathrm{C}$ ) are one of the key technologies for the BepiColombo mission. Details of this aspect of the qualification have been reported $[2,5,6]$. 
Also other electrical components like diodes and resistors were partly modified and tested for the specific mission needs.

\subsection{Coatings and other thermo-optical Surfaces}

For the qualification testing of white coatings, OSRs and Kapton-VDA and Nextel under high temperature conditions in combination with UV/VUV see [5].

\subsection{Reduction of Heat Flux into the Spacecraft}

Obvious design characteristics of the MPO solar array are the measures to reduce the thermal flux into the spacecraft at the interface areas between the solar array and the SC. Both, the yoke-hinge (root-hinge) and the hold down and release mechanisms (HDRMs), are covered by MLI or shields.

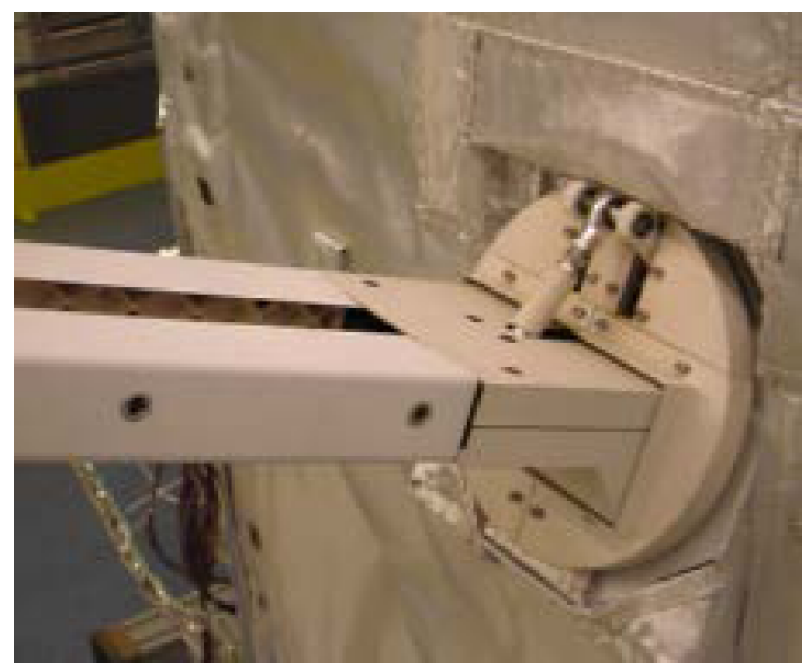

Figure 13. Yoke hinge (root hinge)

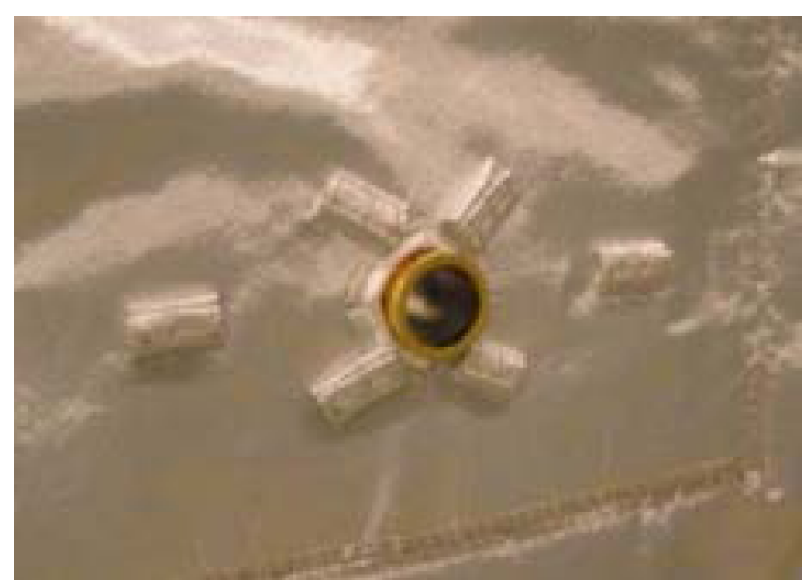

Figure 14. HDRM on SC sidewall under MLI

\section{MPO WING QUALIFICATION}

Beside the continuation of the component qualifications and preparation and testing of qualification-samples and DVT-coupons the wing assembly and testing have been the main tasks for the last months.

\subsection{Thermal vacuum testing}

One of the major wing tests starts already before wing assembly. It is common practice that the thermal vacuum (TV) testing is done on panel level (respectively yoke level). Therefor the panels and the yoke, which are fully equipped with solar cells and OSRs, have been tested for $10 \mathrm{TV}$ cycles from $-170^{\circ} \mathrm{C}$ to $+215^{\circ} \mathrm{C}$. This testing was combined with a bake out to reduce the outgassing of the synthetic materials during the mission.

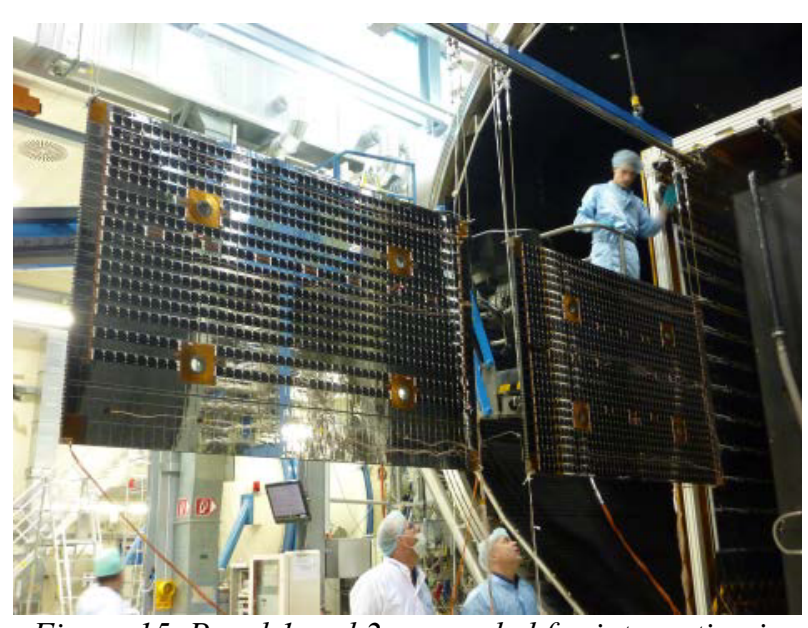

Figure 15. Panel 1 and 2 suspended for integration in the TV chamber

\subsection{Wing deployment tests}

During the launch the wing is folded on the SC side wall. The wing deployment is tested before and after the environmental test program which shall simulate launch conditions (e.g. shaker tests). 


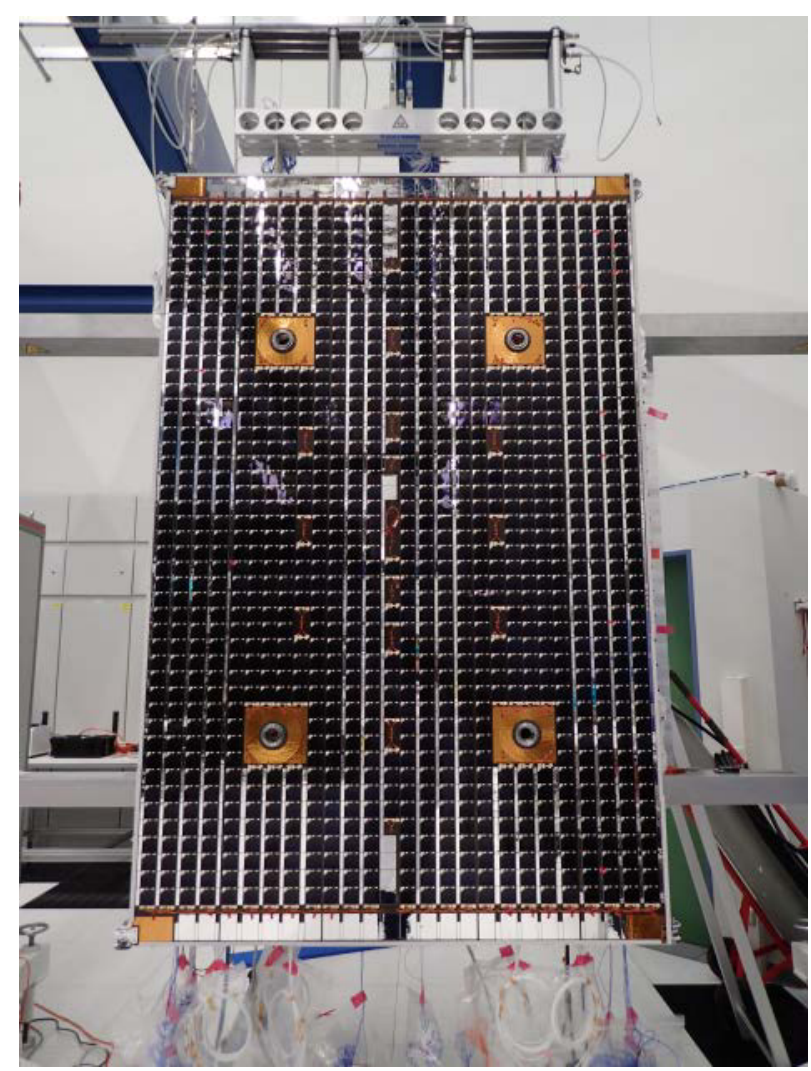

Figure 16. Wing folded to SC sidewall simulator



\subsection{Shaker tests}

By the shaker tests the sine base excitations which are acting on the solar array during launch are simulated. This test is done in 3 axes (1x out-of-plane, $2 \mathrm{x}$ inplane). The structural integrity of the panels and yoke was monitored by ca. 60 accelerometers, 81 strain gauges and 5 load cells.

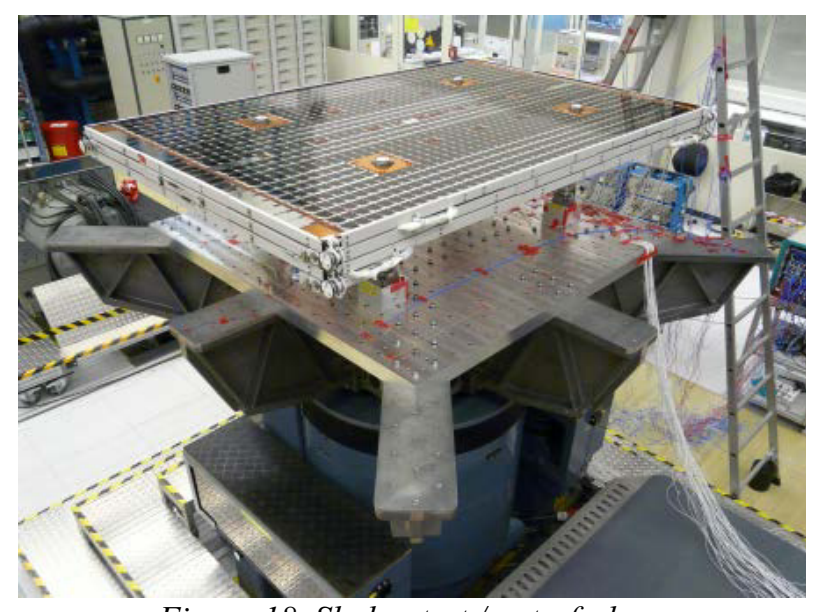

Figure 18. Shaker test / out-of-plane



Figure 19. Shaker test / in-plane

Acoustic tests were performed only on single panel level (EQM panel) and will be performed on system level.

\subsection{Electrical performance and health checks}

The standard electrical characterisations were done before and after environmental testing.

- $\quad$ Power performance (flasher test)

- Electroluminescence (ELM)

- Electrical health checks (insulation, grounding, continuity, etc.) 


\section{SUMMARY}

Fundamental technology developments were needed for the realisation of the BepiColombo MPO solar array. Technology challenges like UV and thermal cycling degradation under extreme temperature conditions were investigated and a new cell design based on the Azur 3 G28 triple junction solar cell was validated under relevant environmental conditions. Due to technology limitations extensive system level analyses have led to design and operations modifications on system level and the MPO solar array. Even with the modifications the environment still imposes quite a challenge on the PVA, solar array structures and other components like high temperature diodes. Dedicated developments were performed in the frame of the project and have been incorporated into the MPO solar array design.

\section{ACKNOWLEDGEMENT}

The work on the Bepi Colombo solar array was carried out under a programme of and funded by the European Space Agency. The view expressed herein can in no way be taken to reflect the official opinion of the European Space Agency.

Besides the authors of this paper a large team has worked on the development of the BepiColombo solar arrays. Contributions of the following colleagues are highly appreciated: C. Zimmermann and C. Noemayr (PVA technologies); J. Müller (design MPO S/A); R. Sebek (design MTM S/A); F. Ustamuijc (structural developments).

The authors express their thanks to AZUR Space Power $\mathrm{GmbH}$, XPERION Aerospace GmbH, W. L. Gore \& Associates GmbH, CNM D+T Microelectronica, GKN Aerospace $\mathrm{GmbH}$ heavily involved into the technology development.

\section{REFERENCES}

1. Petry D., Grünwald. C., Löhberg, A., Brandl, A., Oxynos-Lauschke, C., Andreev, T., van der Ven, R., Schuhmacher, U., Fugger, S., Caon, A., Fiebrich, H.K., (2011). The BepiColombo Mercury Transfer Module and Mercury Planetary Orbiter Solar Array Design and Development. ESPC 2011 - European Space Power Conference.

2. Andreev, T., Zimmermann, C., Löhberg, A., de Jong, S., Schuhmacher, U., Schneider, J., Köstler, W., Parketta, P., Zäh, E., Holzbauer, R., Fink, M., Hall, J., Fernandez Lisbona, E., Lundmark, K., Baur, C., Taylor, S., Panin, F., Caon, A. (2015). Qualifying Solar Array Components for High Intensity, High Temperature Environments - The Test Approach for the Bepi Colombo Solar Arrays, In Proc. 10th European Space Power Conference
ESPC 2014, ESA SP-719 (CD-ROM).

3. T. Hülsheger, C. Brandt, A. Caon, H. K. Fiebrich and T. Andreev, "The Angular Performance Behaviour of Triple Junction Solar Cells“, In 9th European Space Power Conference, ESA SP2102638 (CD-ROM), Saint-Raphaël, France, 2011.

4. Brandt, C., Hülsheger, T., Baur, C., Caon A., Andreev, T., "The Influence of High Light Inclination Angles on the Performance of $\mathrm{GaInP}_{2} / \mathrm{GaAs} / \mathrm{Ge}$ Triple Junction Solar Cells", In: $38^{\text {th }}$ Photovoltaic Specialist Conference (IEEE), Austin, Texas, USA, pp. 1572-1578, June 2011.

5. Nömayr, C., Zimmermann, C., Premysl, J., "Degradation of Solar Array Components in a Combined UV/VUV High Temperature Test Environment, In $11^{\text {th }}$ Space Power Conference ESPC 2016, $3^{\text {rd }}$ to $7^{\text {th }}$ of October 2016, Thessaloniki, Greece

6. Oberhüttinger, C. Quabis, D., Nesswetter, H., Zimmermann, C., "Simulating Large Area, High Intensity AM0 illumination - Test Results from BepiColombo and Solar Orbiter Qualification", In $11^{\text {th }}$ Space Power Conference ESPC 2016, $3^{\text {rd }}$ to $7^{\text {th }}$ of October 2016, Thessaloniki, Greece

7. http://sci.esa.int/bepicolombo/55747-artisticimpression-of-bepicolombo/ 